\title{
Utilidad de la ecografía Doppler color en el seguimiento de la angioplastia carotídea
}

\section{Doppler in Carotid Stent Follow-up}

\author{
Alberto M. Surur ${ }^{1}$ Germán Crespo ${ }^{1} \quad$ Jorge A. Galindez ${ }^{1} \quad$ Lucas Pessini $^{1} \quad$ Marco A. Marangoni ${ }^{1}$ \\ Hugo F. Londero ${ }^{2}$
}

${ }^{1}$ Servicio de Diagnóstico por Imágenes, Sanatorio Allende, Córdoba, Argentina

${ }^{2}$ Servicio de Hemodinamia e Intervenciones por Cateterismo,

Sanatorio Allende, Córdoba, Argentina

Address for correspondence Alberto M. Surur, Servicio de

Diagnóstico por Imágenes, Sanatorio Allende, Córdoba, Argentina

(e-mail: asurur@hotmail.com).

Rev Argent Radiol 2019;83:34-41.

\section{Resumen}

\section{Palabras Clave \\ - ataques cerebrovasculares \\ - estenosis carotídea \\ - ecografía doppler color \\ - angioplastia \\ - stent}

\section{Abstract}

Los ataques cerebrovasculares (ACV), representan un problema mayor en salud y son una causa importante de discapacidad en todo el mundo. La estenosis u oclusión carotídea aterosclerótica causa alrededor del $20 \%$ de las isquemias cerebrovasculares. ${ }^{1}$ Los ACV en los enfermos con estenosis carotídea se producen, en la mayoría de los casos, por la aparición de embolismos distales de trombo formado en la placa, oclusión trombótica aguda debido a rotura de la placa, o bien secundarios a las alteraciones hemodinámicas atribuibles a la estenosis, la cual produce disminución de la perfusión cerebral por el hipoflujo, en casos de estenosis críticas o suboclusivas. El tratamiento de la patología carotídea ha evolucionado de forma considerable a lo largo de los últimos años. Existen dos tratamientos invasivos para la estenosis carotídea significativa, que consisten en el abordaje quirúrgico con endarterectomía o tratamiento por vía percutánea con angioplastia y colocación de stent. En nuestra institución se realizaron, en el periodo comprendido entre marzo de 2013 y junio de 2017, aproximadamente 100 angioplastias carotídeas con colocación de stent. Todas fueron valoradas con ecografía Doppler color (EDC) al mes, a los seis meses y al año (en casos no complicados). En esta revisión bibliográfica es nuestra intención desarrollar las indicaciones, aplicaciones, hallazgos normales y patológicos en el examen de EDC en la evaluación de un paciente con stent carotídeo.

Acute stroke represents a major problem in health and is a major cause of disability worldwide. Atherosclerotic carotid stenosis or occlusion causes around $20 \%$ of cerebrovascular ischemias. ${ }^{1}$ Stroke in patients with carotid stenosis occurs, in most cases, due to embolisms of thrombus formed in the plaque, acute thrombotic occlusion due to rupture of the plaque, or secondary to hemodynamic alterations, attributable to stenosis, which produces decreased cerebral perfusion by low flow, in cases of critical or sub occlusive stenosis. The treatment of carotid disease has evolved considerably over recent years. There are two invasive treatments for significant carotid stenosis, which received

November 8, 2017

accepted

May 13, 2018

published online

July 30, 2018
DOI https://doi.org/

10.1055/s-0038-1660524.

ISSN 1852-9992.
Copyright (c) 2019, Sociedad Argentina de Radiología. Publicado por Thieme Revinter Publicações Ltda., Rio de Janeiro, Brazil. Todos los derechos reservados.

\section{License terms}

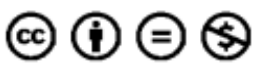



Keywords
- stroke
- carotid stenosis
- doppler ultrasound
- angioplasty
- stent

consist of the surgical approach with endarterectomy or percutaneous treatment with angioplasty and stenting. In our institution, in the period between March 2013 and June 2017, 100 carotid angioplasties with stent placement were performed. All were assessed with color Doppler ultrasound (DUS) at month, six months and one year (in non complicated cases). In this literature review it is our intention to develop the indications, applications, normal and pathological findings in the DUS examination in the evaluation of a patient with carotid stent.

\section{Desarrollo}

Para el estudio de las arterias del cuello existen métodos invasivos y no invasivos. Entre los no invasivos contamos con ecografía Doppler color (EDC), la angiotomografía computada (Angio-TC) y la angiografía por resonancia magnética (Angio-RM). Como método invasivo, la angiografía por sustracción digital (ASD) es el estándar de referencia para el diagnóstico de enfermedad carotídea. El EDC es el método de primera intención. Brinda fundamentalmente información morfológica y hemodinámica. El mismo clasifica las estenosis carotídeas en distintas categorías: normal, menor al $50 \%$, entre 50 y $69 \%$, mayor al $70 \%$, estenosis crítica 0 suboclusiva $(\geq 90 \%)$ y oclusión, de acuerdo a hallazgos morfológicos y hemodinámicos (-Tabla 1). ${ }^{1-3}$

La Angio-TC y la Angio-RM son métodos complementarios, no invasivos, para estudiar la enfermedad carotídea. Además, aportan información anatómica de la porción intracraneal e intratorácica de los vasos del cuello. Ambas se basan en los criterios NASCET para la medición del grado de estenosis y son métodos de elección y complementarios en determinados pacientes (-Figs. 1 y 2 2). ${ }^{4}$ Esos criterios consisten en correlacionar el diámetro de la arteria carótida interna (ACI), en su sitio de máxima estenosis, con el de su segmento distal normal, donde ambas paredes se vuelven paralelas.

El tratamiento para la estenosis carotídea de origen aterosclerótico consiste en la resolución por vía quirúrgica, ya sea endarterectomía o angioplastia, con colocación de stent en pacientes con estenosis significativa (> 70\%), tanto asintomáticos como sintomáticos. ${ }^{4,5}$ Ambos métodos demostraron mejores resultados con respecto al tratamiento médico para este grupo de pacientes. ${ }^{4,6}$ En caso de estenosis no significativas ( $\leq 50 \%$ ) hay amplio acuerdo en la efectividad del tratamiento médico y para estenosis moderadas (50-69\%), está indicado el tratamiento invasivo en caso de placas ateroescleróticas complicadas o en pacientes sintomáticos donde el tratamiento médico ha fracasado. ${ }^{7}$

Varios estudios multicéntricos aleatorizados no encontraron diferencias significativas entre pacientes sometidos a endarterectomía y aquellos a los que se trató con stent, tanto en riesgo de stroke periprocedimiento, infarto de miocardio, muerte e ictus homolateral. ${ }^{8}$ Nuestra institución cuenta con amplia experiencia en angioplastia con colocación de stent para tratamiento de estenosis carotídea por sobre la endarterectomía. El procedimiento angiográfico consiste en canular la arteria carótida, colocar sistemas de protección cerebral contra la embolización, seguido de provocar la dilatación y la colocación de stent. Los stents utilizados en angioplastia carotídea son de tres tipos: stents tubulares expandibles con balón, autoexpandibles de nitinol y tipo malla de acero inoxidable (Wallstent ${ }^{\circledR}$ ). Ese último ofrece una buena cobertura de la placa y distribuye mejor la fuerza radial en la superficie de la lesión evitando el prolapso del material aterosclerótico a través de la malla (-Fig. 3 ). ${ }^{9}$

Para el seguimiento del stent, el EDC es el método de primera intención, por inocuidad, accesibilidad y rapidez. También es útil para el seguimiento, ya que permite detectar la mayoría de las complicaciones siendo el método de elección en esos pacientes. Realizado por exploradores expertos y con los equipos adecuados, el ECD presenta en relación a la ASD sensibilidades superiores al 95\% para la detección o exclusión de estenosis significativa en la ACI con valores predictivos negativos por encima también del $95 \% .^{10}$ Para una adecuada realización se debe seguir un protocolo

Tabla 1 Criterios diagnósticos de estenosis carotídea

\begin{tabular}{|l|l|l|l|l|}
\hline Grado de estenosis & Vps ACl (cm/seg) & $\begin{array}{l}\text { Estimación de } \\
\text { la placa (\%) }\end{array}$ & Ratio Vps ACI/ACC & Vfd ACI (cm/seg) \\
\hline Normal & $<125$ & Sin placa & $<2$ & $<40$ \\
\hline$\leq 50 \%$ & $<125$ & Placa $<50 \%$ & $<2$ & $<40$ \\
\hline $50-69 \%$ & $125-230$ & Placa $>50 \%$ & 2 a 4 & 40 a 100 \\
\hline$\geq 70 \%$ & $>230$ & Placa $>50 \%$ & $>4$ & $>100$ \\
\hline $\begin{array}{l}\geq 90 \%, \text { Critica, } \\
\text { suboclusiva }\end{array}$ & $\begin{array}{l}\text { Alta, baja, } \\
\text { o no detectable }\end{array}$ & Placa con luz visible & Variable & Variable \\
\hline Oclusión & No detectable & Luz no detectable & Ausente & Ausente \\
\hline
\end{tabular}

Abreviaturas: ACC, arteria carótida común; ACl, arteria carótida interna; Vfd, velocidad fin de diástole; Vps, Velocidad pico sistólico. 


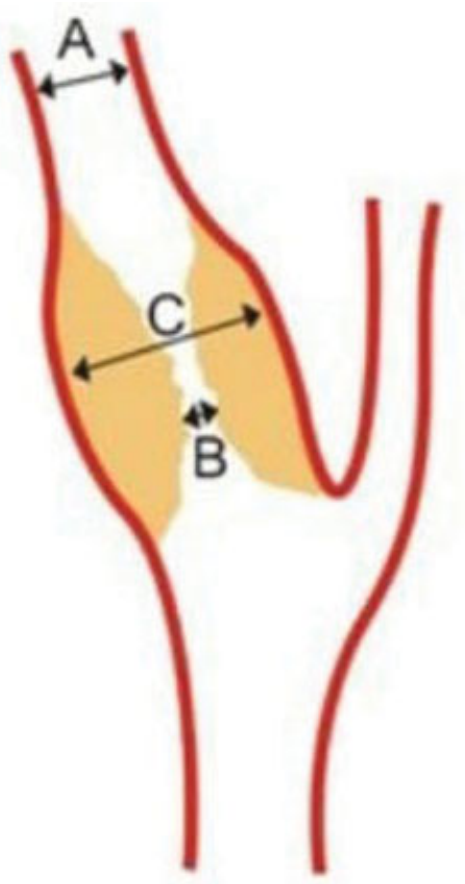

Fig. 1 Método de medición del grado de estenosis, para angiotomografía computada (Angio-TC) y angiografía por resonancia magnética (Angio-RM), según los criterios NASCET. (A) Sector de arteria de calibre normal distal a la estenosis. (B) Sitio de máxima estenosis. (C) Diámetro total de la arteria en el sector de la estenosis.

determinado, evaluando el stent en escala de grises, en modo color y Doppler pulsado (-Esquema 1).

En modo B se obtiene "información morfológica." Ecográficamente el stent es hiperecogénico y puede estar posicionado desde la arteria carótida común en su porción distal o en el nacimiento inmediato de la ACI, con longitudes variables. Es importante registrar la morfología correcta de

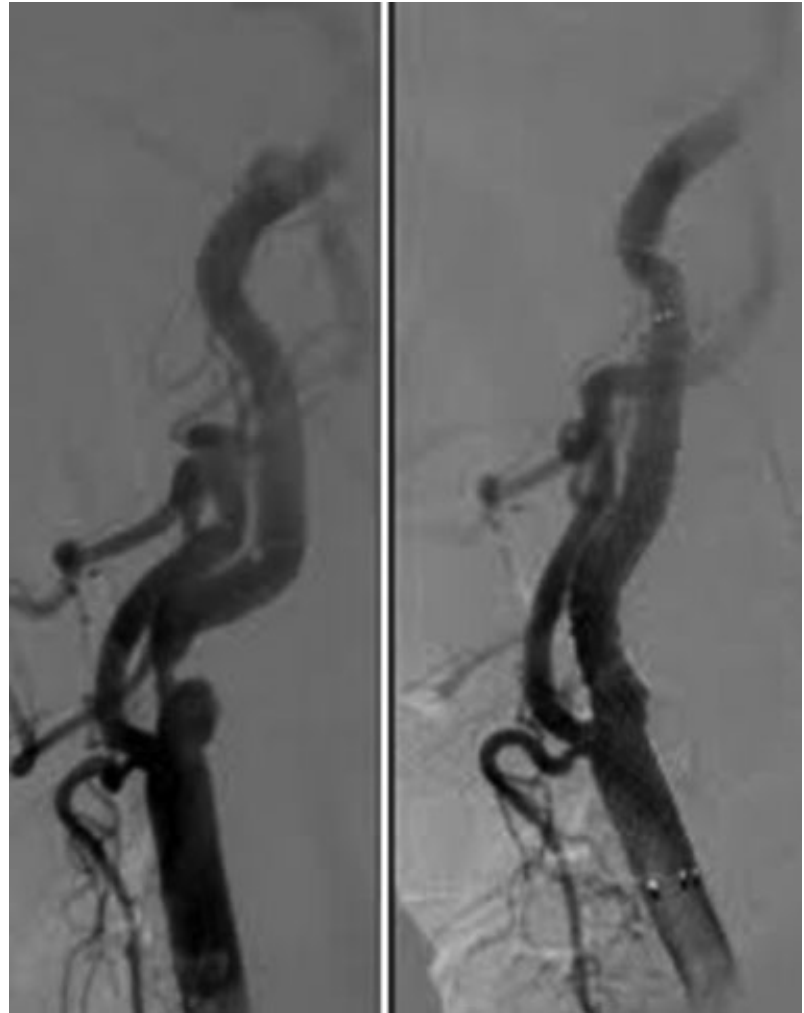

Fig. 3 Angiografía digital donde se observa estenosis significativa de arteria carótida interna (A) e imagen posterior al implante del stent (B).

las paredes de las arterias nativas adyacentes al stent y ver la existencia de placas subyacentes en la zona de estenosis, las cuales pueden provocar una deformidad de la pared del stent y, además, la existencia de placas no cubiertas por el stent. Se debe observar la integridad del mismo y evaluar posibles rupturas (-Fig. 4).
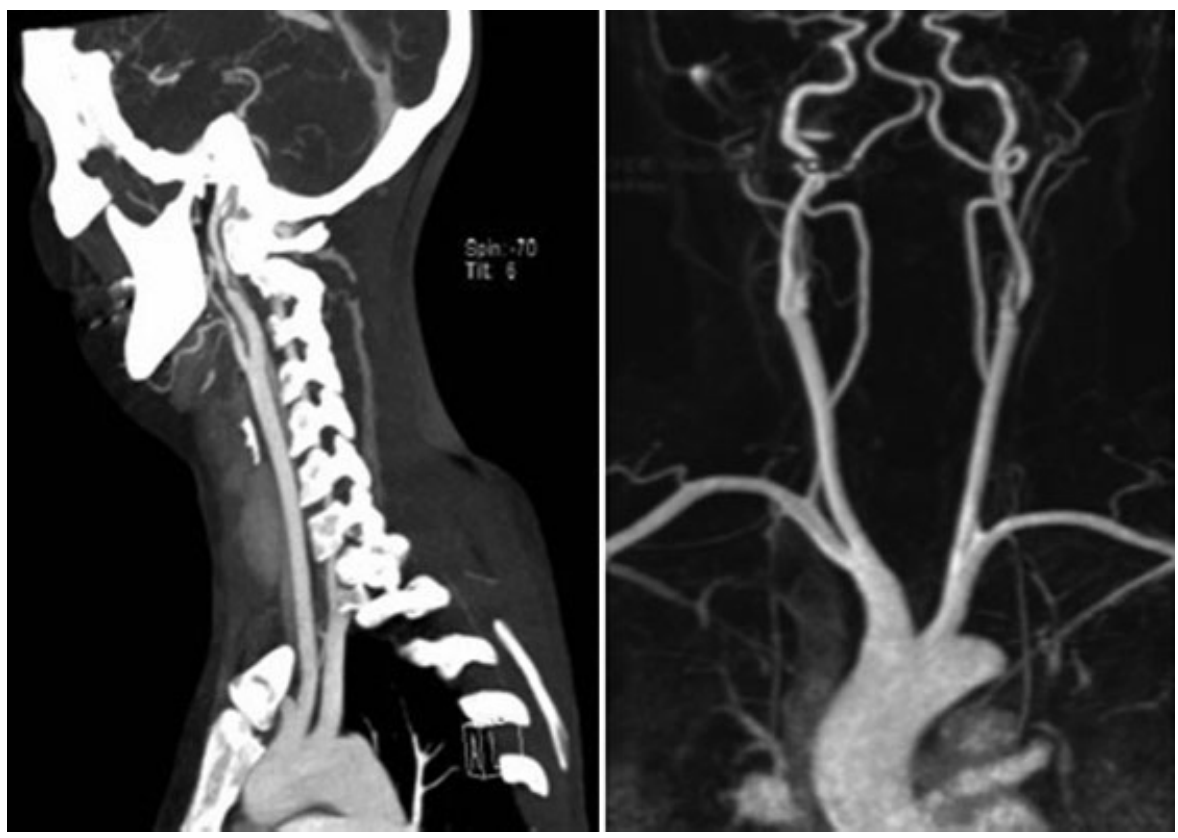

Fig. 2 Angio-TC y Angio-RM de vasos de cuello (A y B). 


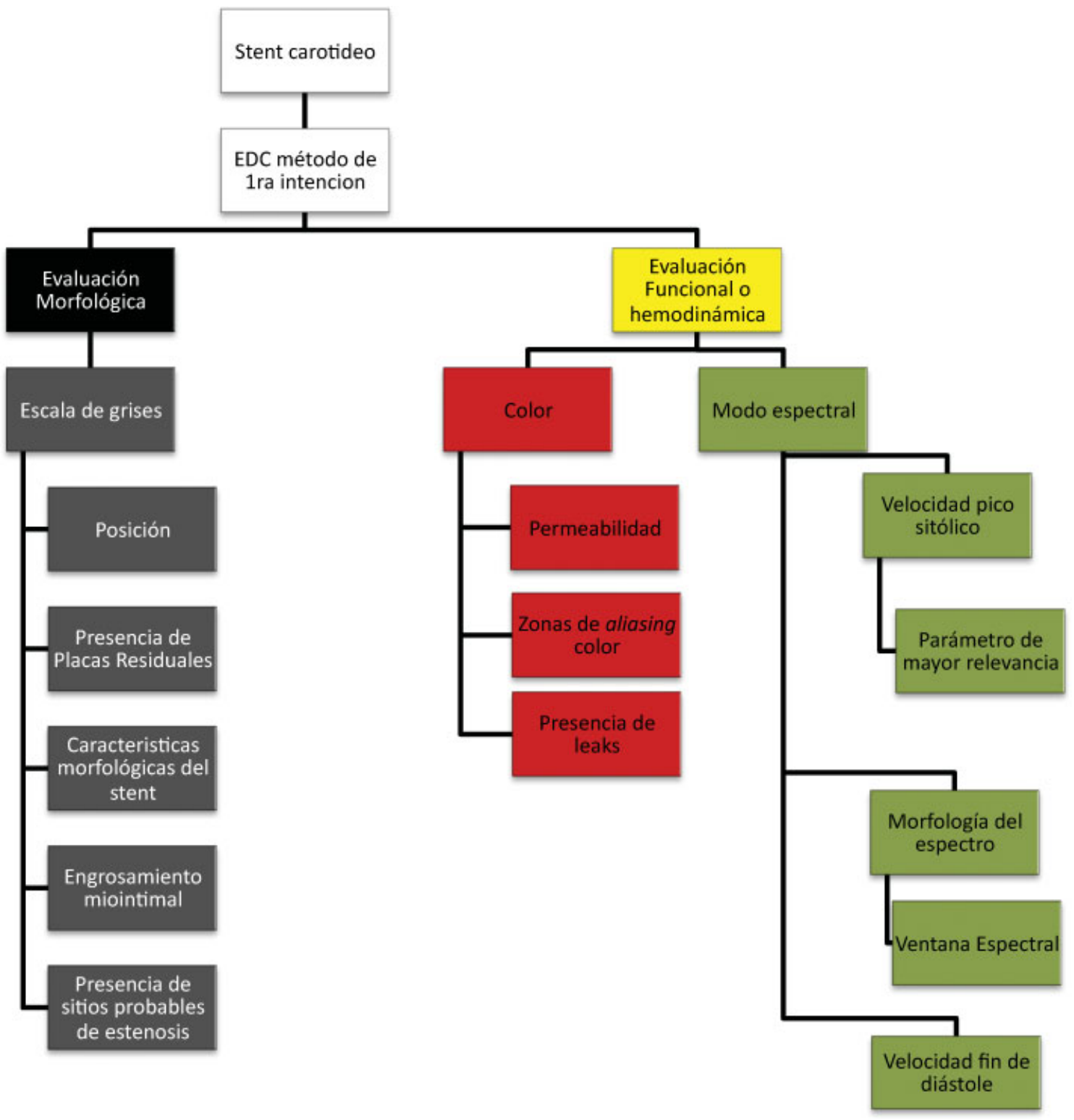

Esquema 1 Pasos a seguir a la hora de evaluar un stent con EDC.

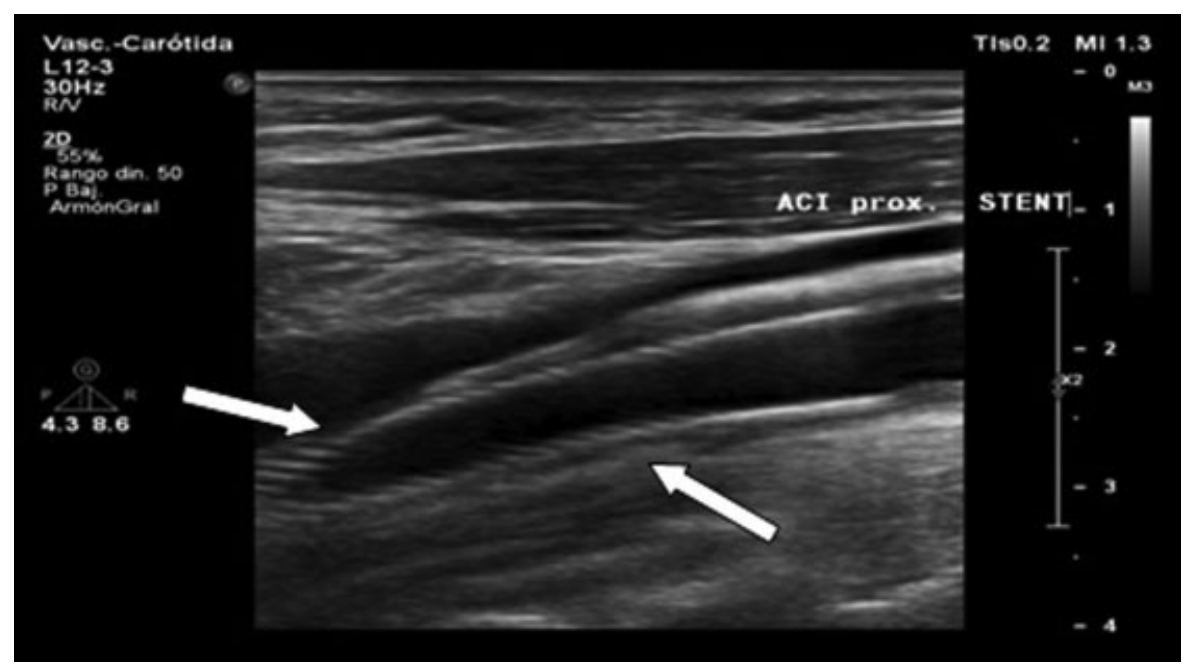

Fig. 4 Ecografía en modo B donde se observa stent con buena posición y aposición a la pared vascular, sin anormalidades en su calibre y luz vascular residual (Flecha). 


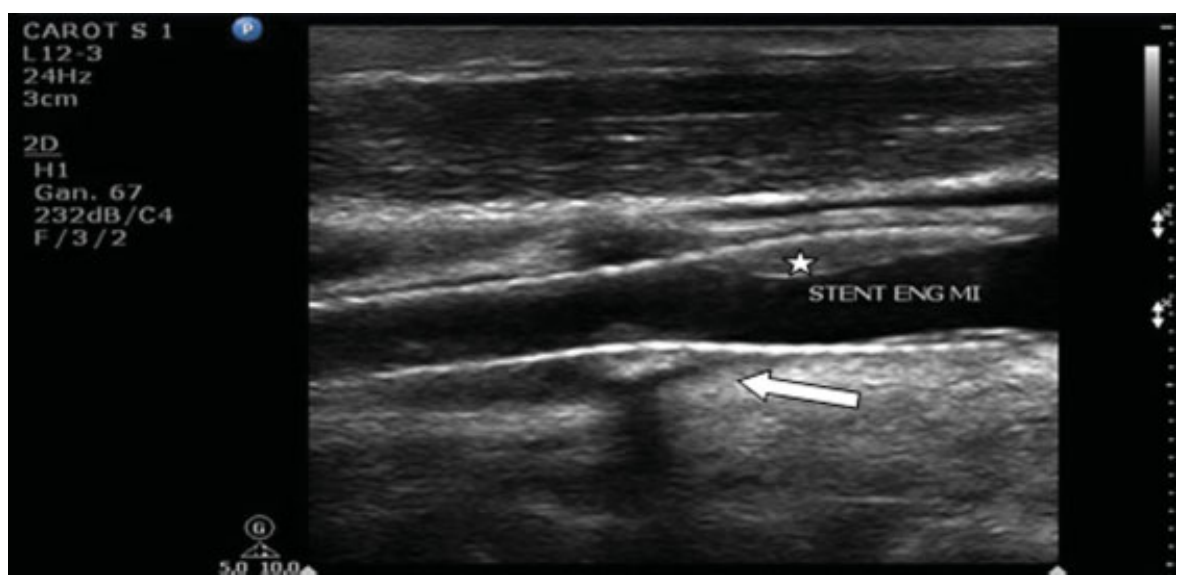

Fig. 5 Ecografía en escala de grises donde se observa la presencia de hiperplasia neointimal (estrella) y placa subyacente al stent con leve deformidad de la pared posterior (flecha).

En caso de estenosis a causa de disección, el tratamiento puede requerir la colocación de dos stent y, por ende, es posible la existencia de desacoples.

En los implantes tipo Wallstent ${ }^{\circledR}$, los más utilizados, cuentan con ciertas desventajas. Una de ellas es la falta de flexibilidad longitudinal, por lo que una vez expandido rectifica la arteria y puede provocar la formación de una angulación de la misma en el extremo distal del stent. Otra desventaja podría ser una mala adapatación a la silueta de la arteria que provoca la formación de espacios muertos entre el stent y la pared arterial por mala aposición, aunque es un hallazgo poco frecuente. ${ }^{9}$ Todos esos hallazgos deben ser observados y descriptos con el EDC.

Otro parámetro a evaluar es la existencia de proliferación neointimal hacia la luz del stent, la cual tiende a aumentar de grosor en los primeros 3, 6 y 12 meses, para luego estabilizarse hacia los 24 meses o evolucionar y terminar provocando una reestenosis. ${ }^{11}$ Se considera que una respuesta biológica exagerada ante la injuria vascular es responsable de ello. Dicha hiperplasia se observa en escala de grises como una línea isoecogénica o hipoecóica, la cual prolifera entre la superficie interna del stent y el lumen
(- Fig. 5). ${ }^{12}$ Sitios probables de reducción de la luz vascular son observados en escala de grises.

En modo color se debe documentar la permeabilidad del stent, la superficie del mismo y de la pared adyacente. Es preciso objetivar la existencia de zonas de aliasing color. Eso sucede debido a una pérdida de la laminaridad del flujo o bien a la existencia de zonas de turbulencia como consecuencia de una reducción de la luz vascular y, por lo tanto, es un hallazgo de importancia, ya que sería el sitio de interés para evaluar con el Doppler espectral en busca de estenosis (-Fig. 6). Frecuentemente, existen estenosis residuales de las arterias carótidas externas, secundarias al implante de la prótesis, sin implicancias patológicas relevantes.

Por último, debemos también evaluar la presencia de leaks, aunque de presentación poco frecuente con los stents tipo Wallstent ${ }^{\circledR}$. Eso generalmente sucede debido a una mala aposición del stent, quedando un pasaje de flujo entre la pared vascular y el stent.

Uno de los mayores temores, luego de la angioplastia, es la reestenosis, así como la oclusióndel stent. Algunos reportes mencionan una baja tasa de reestenosis luego de la angioplastia. $^{13}$ Lal y col., demuestran, en un estudio

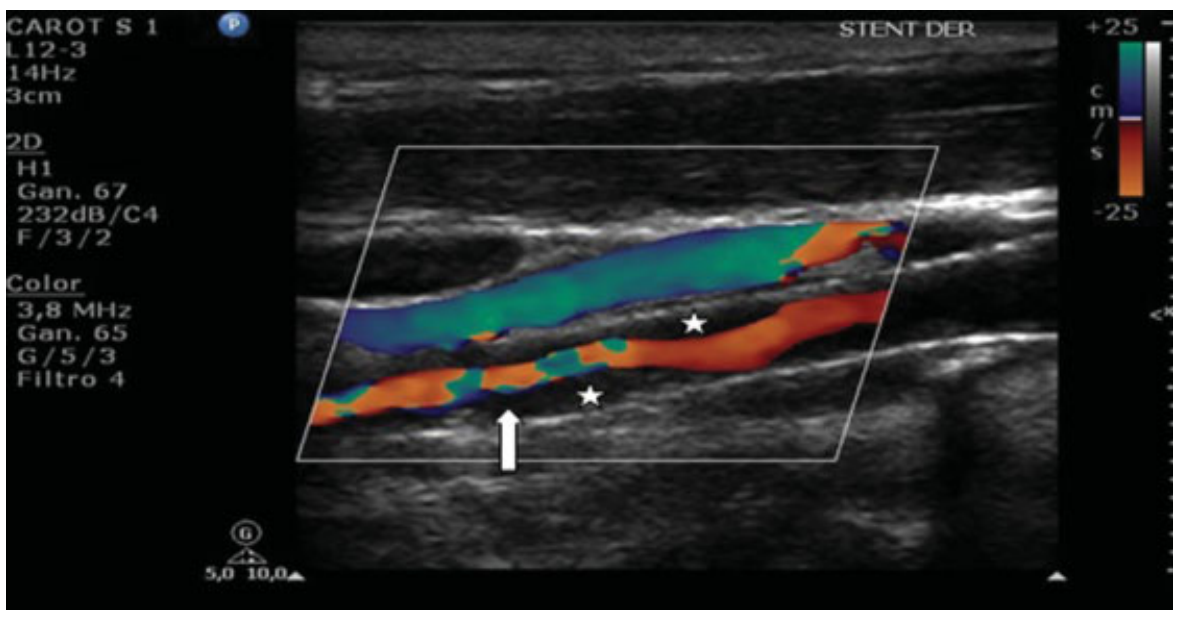

Fig. 6 Ecografía Doppler color (EDC) con ocupación de un tejido hipoecóico que prolifera hacia la luz (hiperplasia neointimal), con reducción de la luz vascular (estrella). El mapa color muestra el lumen disminuido con una zona de aliasing color (flecha). 
randomizado, estenosis significativa mayor al 70\% u oclusión solo en 58 pacientes sobre un total de 1.086 , todos en seguimiento con ECD, en controles sucesivos a los $1,6,12$, 24 y 48 meses. $^{14}$

$\mathrm{Al}$ igual que las estenosis de arterias nativas, en los stent se tienen en cuenta los mismos parámetros técnicos. Consiste en medir la reducción del diámetro de la luz, a nivel del diámetro menor, con respecto al diámetro mayor dentro del stent o de la arteria nativa pos stent, y realizar estudio en modo espectral obteniendo el valor de velocidad de fin de diástole (VFD) y velocidad pico sistólico (VPS), siendo éste ultimo el parámetro de mayor relevancia. Para ello se posiciona el transductor sobre el eje longitudinal del vaso, con un ángulo Doppler de $60^{\circ}$, recorriendo con el volumen de muestra toda la extensión de la arteria, buscando la zona con mayor VPS y así obtener registros reproducibles. ${ }^{15}$

En el caso del stent, el estudio en modo espectral debe ser realizado pre stent, intra stent en su sector proximal, medio y distal y posterior al mismo. Es necesario tomar en cuenta las características morfológicas de la onda. Debido a la laminaridad de los glóbulos rojos en el vaso nativo la onda del Doppler se agrupa en la región superior del espectro, dejando por debajo un área vacía, la cual se denomina ventana espectral ( - Fig. 7). El flujo en el interior del stent, en algunos casos, puede perder esa uniformidad por lo que implica una pérdida de la ventana espectral y no significar un hallazgo patológico. Además se puede objetivar ausencia de la ventana espectral en casos de estenosis, a causa del flujo turbulento.

Cuando existen zonas sospechosas de reestenosis el estudio en modo espectral debe obtener registros en el sitio previo a la estenosis, dentro de la misma correspondiente con la zona de máximo aliasing, y posterior a ella, evaluando las distintas morfologías de onda y obteniendo la VPS (-Fig. 8).

En los pacientes con stent, los criterios de VPS deben ser corregidos. Algunos reportes indican que luego de la angioplastia carotídea se altera la compliance del vaso nativo por lo cual varían las velocidades. Hay acuerdo en que las mismas se ven aumentadas, lo cual tiende a confundir y a sobreestimar la estenosis. En el análisis de Ringer y col., se

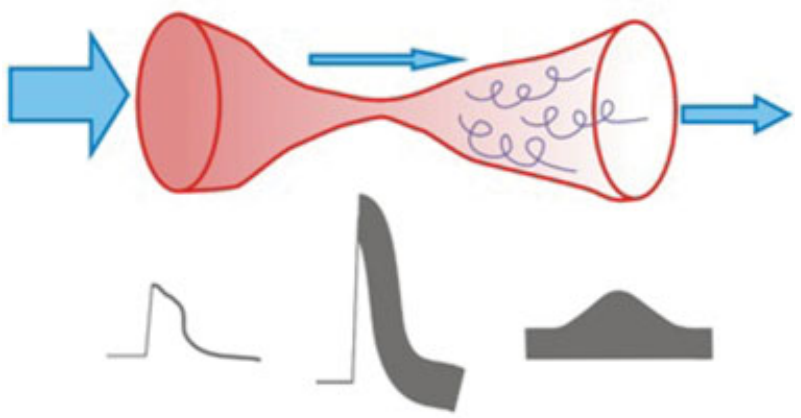

Fig. 8 Representación esquemática donde se observan las diferentes morfologías de onda. En el sector previo a la estenosis, donde el flujo es laminar, se obtienen morfologías de onda normales con conservación de la ventana espectral (flecha). En el sitio de la estenosis la onda adquiere alta velocidad y pérdida de la ventana espectral (estrella). Finalmente posterior a la estenosis se observa un flujo de tipo turbulento, con baja velocidad y disminución del tiempo de aceleración (cabeza de flecha).

evaluaron los pacientes siete días posterior a la colocación de stent, usando el método estándar de VPS, encontrando sobre un total de 114 pacientes, 40 con velocidades elevadas, con criterios de reestenosis, que posteriormente en control angiográfico no tuvieron evidencia de estenosis mayor al $50 \%{ }^{16}$

Múltiples estudios establecieron criterios de VPS óptimos a la hora de evaluar posibles reestenosis. AbuRahma y col., establecen como punto de corte de VPS en, $>154 \mathrm{~cm} /$ seg > 30\%; > $224 \mathrm{~cm} / \mathrm{seg}>50 \%$; > $325 \mathrm{~cm} / \mathrm{seg}>80 \%{ }^{17}$

Stanziale y col. reportan resultados similares, determinando como estenosis mayor al 50\% un punto de corte de VPS de $230 \mathrm{~cm} / \mathrm{seg}$ y mayor al 70\% un pico sistólico de $350 \mathrm{~cm} / \mathrm{seg}$, con una especificidad mayor al 95\% para ambos. ${ }^{18}$ Esa última es la escala que utilizamos en nuestra institución (-Fig. 9).

Zhou W y col., en cambio, definen como criterio fiable para el diagnóstico de reestenosis superiores al $70 \%$ un pico sistólico de $300 \mathrm{~cm} / \mathrm{seg} .{ }^{19}$ En caso de oclusión del stent se debe documentar su existencia. Para ello empleamos los

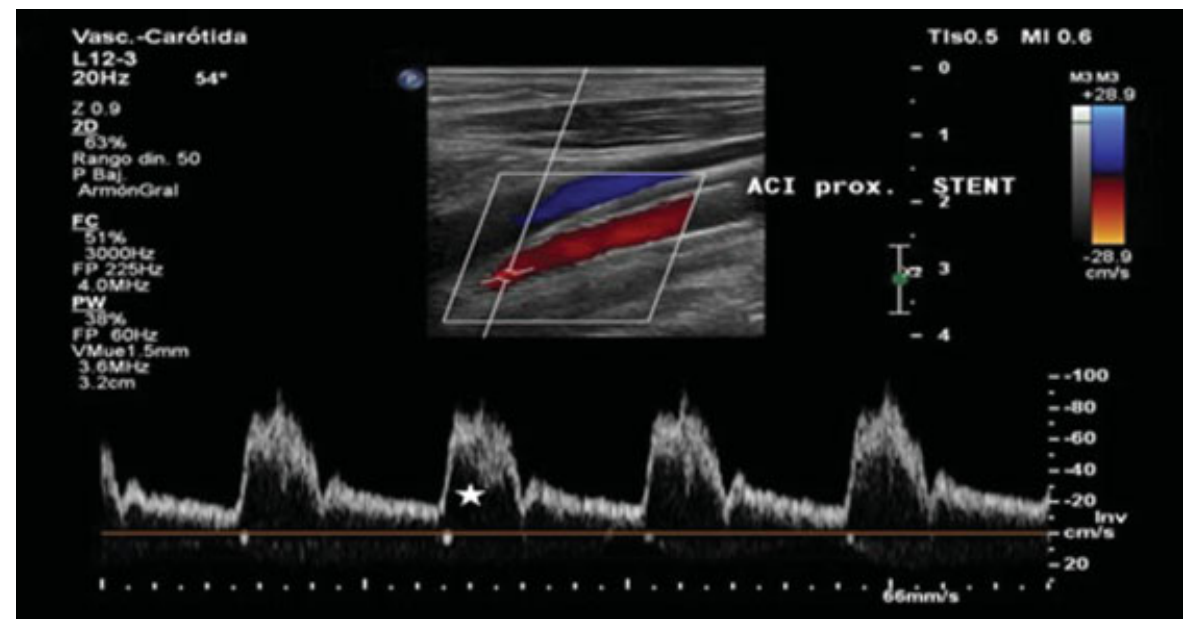

Fig. 7 EDC y espectral en la porción media de un stent con ondas de morfología y velocidad normal (estrella, ventana espectral). 


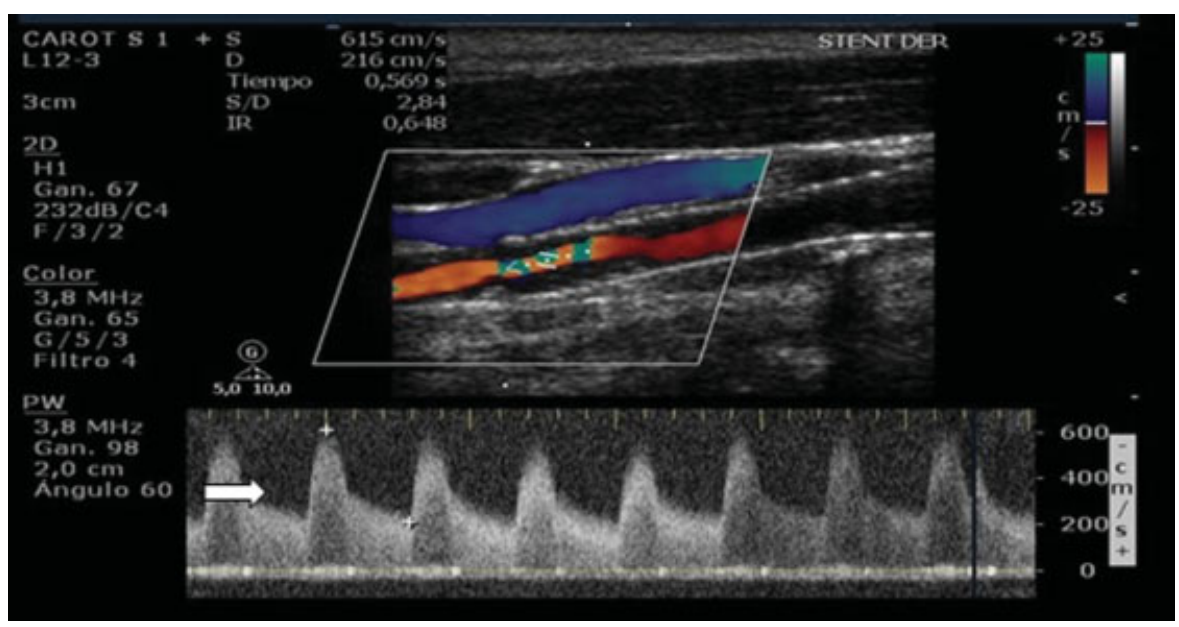

Fig. 9 El EDC detectó una zona de aliasing y en el modo espectral se observa flujo turbulento, tipo "jet," con pérdida de la ventana espectral y se registró VPS y VFD $(615 \mathrm{~cm} / \mathrm{seg}$ y $216 \mathrm{~cm} / \mathrm{seg})$, comportándose como una estenosis significativa > 70\% según Stanziale y col.

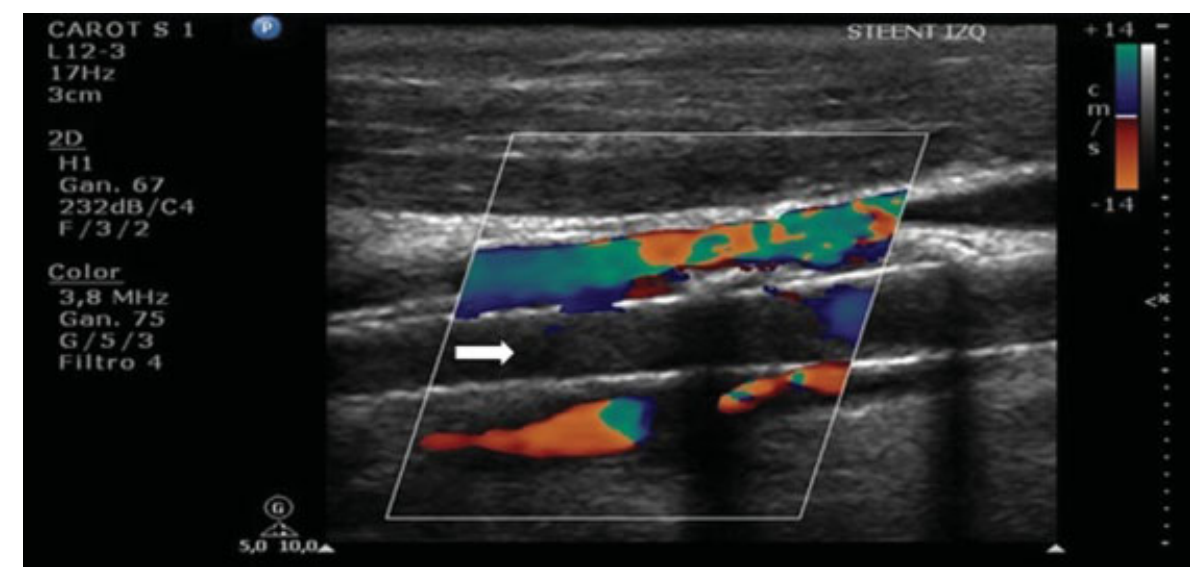

Fig. 10 EDC donde se observa ausencia de flujo en el mapa color dentro del stent (flecha).

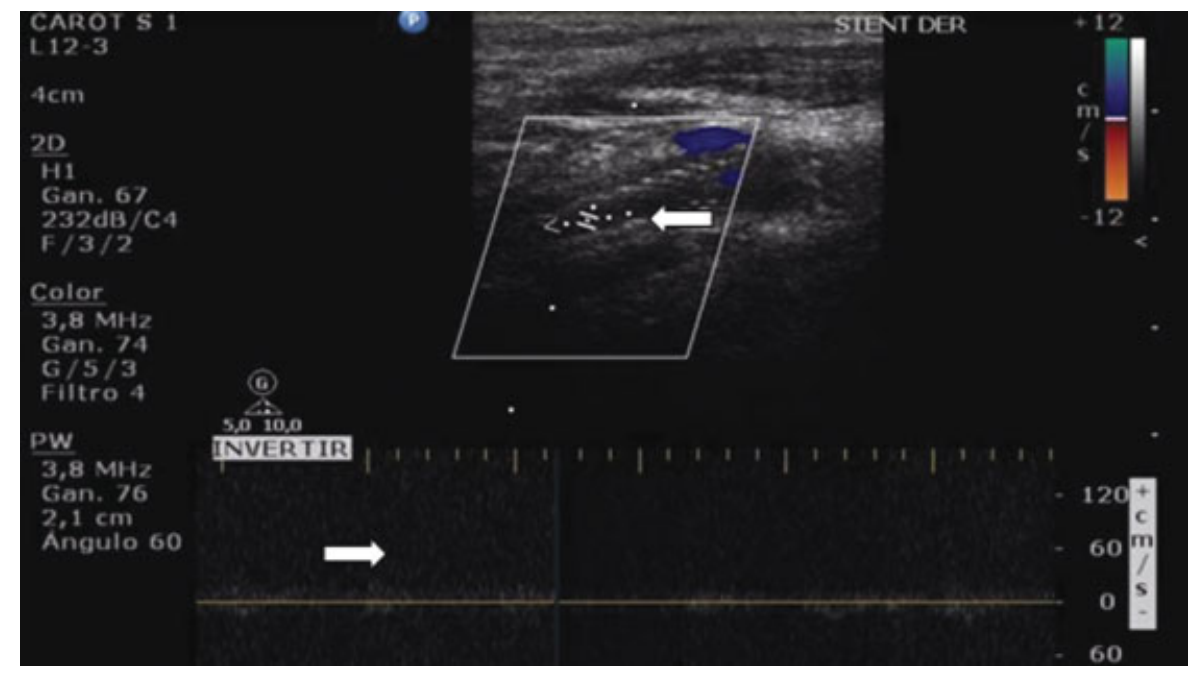

Fig. 11 EDC y espectral donde se observa ausencia de flujo tanto en el mapa color como en el Doppler espectral (flechas).

mismos criterios, realizando registros en modo B, Doppler color y espectral. En las obstrucciones agudas se observa la presencia de un trombo hipoecoico, que ocupa la totalidad de la luz vascular, y de aspecto globuloso. Hacia la fase crónica aumenta la ecogenicidad del trombo y disminuye el calibre del vaso. Por último, la oclusión se va a manifestar con ausencia de flujo tanto al examen Doppler color como espectral (-Figs. 10 y 11). 


\section{Conclusión}

La ecografía Doppler color (EDC) constituye uno de los métodos de elección para la evaluación de los stent carotídeos. Por ese motivo, resulta importante esta revisión, la cual demuestra sus hallazgos normales y patológicos, logrando una adecuada valoración de pacientes con stent carotídeo. Así podrán obtenerse los mejores resultados del método y disminuir sus errores.

\section{Responsabilidades Éticas}

Protección de personas y animales. Los autores declaran que para esta investigación no se han realizado experimentos en seres humanos ni en animales.

Confidencialidad de los datos. Los autores declaran que han seguido los protocolos de su centro de trabajo sobre la publicación de datos de pacientes.

Derecho a la privacidad y consentimiento informado. Los autores declaran que en este artículo no aparecen datos de pacientes.

\section{Conflicto de Intereses}

Los autores declaran no tener ningún conflicto de intereses, con excepción del Dr. Surur que declara como posible conflicto de interés ser Editor Asociado de la RAR.

\section{Bibliografía}

1 Liapis CD, Bell PR, Mikhailidis D, et al; ESVS Guidelines Collaborators. ESVS guidelines. Invasive treatment for carotid stenosis: indications, techniques. Eur J Vasc Endovasc Surg 2009;37(4, Suppl):1-19

2 Surur AM, Buccolini TV, Londero HF, Marangoni MA, Allende NJ. Valoración no invasiva de la estenosis carotídea de causa aterosclerótica: correlación entre la ecografía Doppler color y la angiografía por resonancia magnética con gadolinio. Rev Argent Radiol 2013;77(04):267-274

3 Grant EG, Benson CB, Moneta GL, et al. Carotid artery stenosis: grayscale and Doppler US diagnosis-Society of Radiologists in Ultrasound Consensus Conference. Radiology 2003;229(02):340-346

4 Ferguson GG, Eliasziw M, Barr HW, et al. The North American Symptomatic Carotid Endarterectomy Trial : surgical results in 1415 patients. Stroke 1999;30(09):1751-1758

5 MRC European Carotid Surgery Trial: interim results for symptomatic patients with severe (70-99\%) or with mild (0-29\%) carotid stenosis. European Carotid Surgery Trialists' Collaborative Group. Lancet 1991;337(8752):1235-1243

6 Sociedad Argentina de Cardiología, Sociedad N Argentina. Consenso de estenosis carotídea. Rev Argent Cardiol 2006;74:160-174

7 Halliday A, Harrison M, Hayter E, et al; Asymptomatic Carotid Surgery Trial (ACST) Collaborative Group. 10-year stroke prevention after successful carotid endarterectomy for asymptomatic stenosis (ACST-1): a multicentre randomised trial. Lancet 2010;376(9746):1074-1084

8 Brott TG, Howard G, Roubin GS, et al; CREST Investigators. LongTerm Results of Stenting versus Endarterectomy for CarotidArtery Stenosis. N Engl J Med 2016;374(11):1021-1031

9 Londero HF. Martínez Riera, Paoletti FE, Surur AM, Martín JJ. Intervención percutánea carotídea: aspectos técnicos y resultados. En: Sousa A, Abizaid A, Martínez Ríos M, Berrocal D, Sousa JE, eds. Intervenciones Cardiovasculares - SolaCl. Bogotá: Distribuna Editorial; 2015:115-25

10 Fortuño JR, Perendreu J, Falco J, Canovas D, Branera J. Estenosis carotídea: cómo se diagnostica y se trata adecuadamente. Radiologia 2006;48(03):119-136

11 Willfort-Ehringer A, Ahmadi R, Gschwandtner ME, et al. Healing of carotid stents: a prospective duplex ultrasound study. J Endovasc Ther 2003;10(03):636-642

12 Willfort-Ehringer A, Ahmadi R, Gruber D, et al. Arterial remodeling and hemodynamics in carotid stents: a prospective duplex ultrasound study over 2 years. J Vasc Surg 2004;39(04):728-734

13 Chung J, Valentine W, Sharath SE, et al. Percutaneous intervention for carotid in-stent restenosis does not improve outcomes compared with nonoperative management. J Vasc Surg 2016;64 (05):1286-1294.e1

14 Lal BK, Beach KW, Roubin GS, et al; CREST Investigators. Restenosis after carotid artery stenting and endarterectomy: a secondary analysis of CREST, a randomised controlled trial. Lancet Neurol 2012;11(09):755-763

15 Robbin ML, Lockhart ME, Weber TM, et al. Carotid artery stents: early and intermediate follow-up with Doppler US. Radiology 1997;205(03):749-756

16 Ringer AJ, German JW, Guterman LR, Hopkins LN. Follow-up of stented carotid arteries by Doppler ultrasound. Neurosurgery 2002;51(03):639-643, discussion 643

17 AbuRahma AF, Abu-Halimah S, Bensenhaver J, et al. Optimal carotid duplex velocity criteria for defining the severity of carotid in-stent restenosis. J Vasc Surg 2008;48(03):589-594

18 Stanziale SF, Wholey MH, Boules TN, Selzer F, Makaroun MS. Determining in-stent stenosis of carotid arteries by duplex ultrasound criteria. J Endovasc Ther 2005;12(03):346-353

19 Zhou W, Felkai DD, Evans M, et al. Ultrasound criteria for severe in-stent restenosis following carotid artery stenting. J Vasc Surg 2008;47(01):74-80 